\title{
Neural Correlates of Attentive Selection for Color or Luminance in Extrastriate Area V4
}

\author{
Brad C. Motter \\ VA Medical Center, and Departments of Physiology and Neurosurgery, SUNY Health Science Center, Syracuse, New \\ York 13210
}

\begin{abstract}
Rhesus monkeys were trained on a conditional orientation discrimination task in order to assess whether attentive selection for a color or luminance stimulus feature would affect visual processing in extrastriate area $\mathrm{V} 4$. The task required monkeys to select a bar stimulus based on its color or luminance and then to discriminate the angular tilt of the selected stimulus. The majority of neurons $(74 \%)$ were selectively activated when the color or luminance of the stimulus in the receptive field matched the color or luminance of the cue. The activity was attenuated when there was not a match between the stimulus and the cue. The differential activation was based on the presence or absence of the stimulus feature and was independent of spatial location. Across the population of $\mathbf{V} 4$ neurons, optimal stimuli that matched the selected color or luminance elicited about twice the activity as stimuli that did not match the selected feature. The feature-selective changes in activity were observed to develop beginning about $200 \mathrm{msec}$ after the stimulus onset and were maintained over the remainder of the behavioral trial. In this task the activity of V4 neurons reflected a selection based on the cued feature and not simply the physical color or luminance of the receptive field stimulus. Under these conditions, the topographic representation of the neural activity in area $\mathbf{V} 4$ highlights the potential targets in the visual scene at the expense of background objects. These observations offer a physiological counterpart to psychophysical studies suggesting that stimuli can be preferentially selected in parallel across the visual field on the basis of a unique color or luminance feature.
\end{abstract}

[Key words: visual search, preattentive vision, color, area V4, single unit, attention, conditional discrimination]

Investigations of visual processing in area $\mathrm{V} 4$ have provided a number of striking findings relating behavioral relevance to neuronal activity. Previous studies have looked at the effects of attention on the processing of stimulus properties in terms of either the enhanced processing of stimulus features (Haenny and Schiller, 1988; Haenny et al., 1988; Spitzer et al., 1988; Maunsell et al., 1991) or the limitation of processing to small areas during focal attentive tasks (Moran and Desimone, 1985; Motter, 1993). This report addresses a different aspect of attention in V4, name-

\footnotetext{
Received June 25, 1993; revised Sept. 13, 1993; accepted Sept. 29, 1993.

This work was supported by the VA Medical Research program.

Correspondence should be addressed to Brad C. Motter, Research Service 151 , VA Medical Center, 800 Irving Avenuc, Syracusc, NY 13210.

Copyright (C) 1994 Society for Neuroscience $0270-6474 / 94 / 142178-12 \$ 05.00 / 0$
}

ly, the selection of stimuli based on their possession of a particular stimulus feature, in this case color or luminance.

One of the simplest cognitive acts we perform is the selection of relevant information from our cluttered visual environment. The selective task is enormous, yet we do it with remarkable efficiency. Psychophysical experiments have suggested that early in visual processing the incoming information is sorted and grouped according to the similarity of simple surface features, such as color, luminance, or texture, or simple shape features, such as orientation or size (Treisman and Gelade, 1980; Julesz, 1981; Cavanagh et al., 1990). These sets of features may be organized into separate topographic representations or maps. This segmentation of the scene into what are essentially feature maps is considered to occur in parallel across the visual scene and to occur preattentively or at lcast bcfore focal attentive processes take place (Treisman, 1982; Bergen and Julesz, 1983). Objects whose locations do not "pop out" in the feature maps by their possession of unique features are found by an active focal attentive search that can be drawn or guided to locations in the feature maps that contain matches with prominent features of the goal object (Wolfe et al., 1989). The focal attentive process may serve to bind together feature information from different maps at each spatial location attended (Treisman and Schmidt, 1982).

The separate feature maps indicated psychophysically invite a comparison with the physiological feature sensitivities and retinotopic maps that exist in primary and extrastriate visual areas. Physiological and anatomical studies have supported the idea that early visual cortical areas provide a general low-level processing of visual information that is then distributed into a hierarchy of visual pathways that are selectively engaged depending upon the processing requirements (Desimone et al., 1985; Van Essen, 1985; Maunsell and Newsome, 1987). Clinical reports of rather specific visual feature defects that result from discrete brain lesions have given support to the distributed processing concept (Meadows, 1974; Damasio et al., 1980; Zeki, 1990; Regan et al., 1992). Human studies using positron emission tomography not only have supported this concept (Petersen et al., 1988; Posner et al., 1988; Zeki et al., 1991), but also have provided evidence that different attentive strategies selectively engage different parts of the visual system (Corbetta et al., 1991). This suggests that higher-order processes dynamically create and govern linkages between various brain areas for the purpose of solving different types of perceptual challenges.

Area $\mathrm{V} 4$ has received particular interest because of its position in the chain of pathways leading to the temporal lobe and because of physiological studies suggesting that $\mathrm{V} 4$ has an important role in the process of color perception (Zeki, 1973, 1983). 
In addition to studies that have quantitatively assessed the response sensitivities of V4 neurons to color and form dimensions (Desimone and Schein, 1987; Schein and Desimone, 1990), other physiological studies have demonstrated several neural correlates of focal attentive processing within area V4 (Moran and Desimone, 1985; Haenny and Schiller, 1988; Haenny et al., 1988; Spitzer et al., 1988; Maunsell et al., 1991; Motter, 1993). However, relatively little emphasis has been given to defining the neural correlates of feature-selective processes that may be guiding the selection of candidate targets. This may be due in part to the notion that such processes are automatic and are represented by the basic response sensitivities of neurons.

The aim of the present study was to examine whether the selection of a particular stimulus feature produced changes in the neural responses to stimuli containing that feature. Experiments were designed to compare the neural activity evoked by identical stimuli under different conditions of feature selection. One of the design problems encountered in studying events in the interval just before focal attentive processing is the briefness of the entire selection process when subjects are confronted with relatively simple search displays. In order to prolong the interval during which the prefocal attentive processes might be observed, a two-stage conditional orientation discrimination (CD) task was developed. Feature-selective processes are examined during an interval in which the color or luminance of the cue defines a subset of stimuli that must contain the target stimulus, but the final selection and discrimination of the target stimulus is delayed until an unambiguous choice is revealed.

Some of these results have been described in a preliminary report (Motter, 1992).

\section{Materials and Methods}

Behavioral paradigms. Three rhesus monkeys were trained on a twostage conditional orientation discrimination $(\mathrm{CD})$ task that required them to select a bar stimulus based on its color or luminance and then to discriminate the angular tilt of the selected stimulus. They first fixated a small circular fixation spot whose color or luminance provided the conditional cue for the trial. After establishing fixation of the spot and signaling readiness by pulling back a behavioral key, they were next presented with an array of four or six stimulus bars composed of combinations of two orientations and two colors or luminances (Fig. 1). During the array presentation the subject could select a subset of bar stimuli that matched the color or luminance of the fixation spot. However, because of the multiple color matches, a final orientation judgement could not be made or predicted until the array was reduced to just two stimuli, only one of which matched the fixation cue color or luminance. The full array lasted for a variable period of time (1500$2700 \mathrm{msec}$ ), and was then reduced to two stimuli for $200 \mathrm{msec}$ before being blanked out entirely. The monkey made its orientation judgement of the matching bar stimulus by pushing either a righthand button for rightward tilts of the bar stimulus or a lefthand button for leftward tilts. Steady fixation of the central fixation spot without eye blinks and eye movements to peripheral sites was required during the entire stimulus sequence. An infrared corneal reflection oculometer was used to monitor eye position (Motter and Poggio, 1984).

Prior to studying each neuron in the conditional discrimination paradigm, the neuron's receptive field was located and mapped and the response preferences for stimulus size, color, and orientation were determined using a simple orientation discrimination paradigm. This paradigm required the monkey to ignore peripheral stimuli while fixating a central spot and then to make an orientation discrimination of a small bar flashed directly over the fixation spot (see Fig. 1).

Stimulus presentation. Stimuli were generated using a Raster Technologies model $1 / 10$ display unit hosted by a PDP-11/34 computer and were displayed on a Mitsubishi C6922 monitor located $60 \mathrm{~cm}$ in front of the monkey. Stimulus timing and eye position sampling were synchronized to the $60 \mathrm{H} z$ frame rate of the display system. The time of occurrence of those events and the neural activity were recorded with

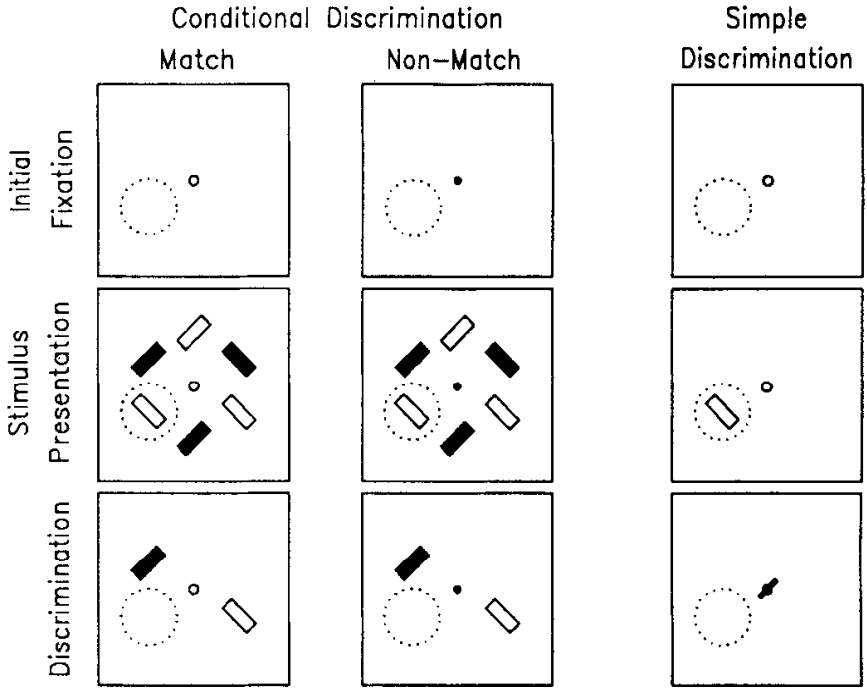

Figure 1. Behavioral paradigms. Each framc represents a field of view, with the fixation spot in the center and the neuron's receptive field indicated by the dotted circle. For either task the subject initially fixates the sinall fixation spot, is then presented with an array of stimuli (left) or a single stimulus (right), and then in a final period must make an orientation discrimination. For the conditional discrimination task on the left the discrimination is made when the array is reduced to two bar stimuli, and the monkey reports the orientation of the remaining bar that matches the fixation spot in color. Match and nonmatch conditions are distinguished by whether or not a color or luminance match occurs between the fixation spot and the stimulus in the receptive field during the stimulus presentation period. For the SD task on the right the monkey ignores peripheral stimulus presentations and makes an orientation discrimination of a bar flashed directly over the fixation spot. The stimuli and their locations in the array are randomized from trial to trial. Note that in the conditional discrimination tasks the receptive field stimulus is not necessarily one of the two stimuli remaining at the end of the trial. The figure is not scaled proportionately.

a precision of $100 \mu \mathrm{sec}$. Each series of stimulus conditions used a pseudorandomized sequence of stimulus selection so that the monkey could not predict stimulus selection or target location. The stimuli consisted of rectangular bars of various sizes, orientations, and colors presented against a background gray of $7.5 \mathrm{~cd} / \mathrm{m}^{2}$. The fixation target was a small filled circle $\left(0.2^{\circ}\right)$. Stimulus arrays contained either four or six bar stimuli equally spaced along an imaginary circle centered on the fixation spot. The array was placed so that one bar stimulus was centered in the receptive field of the neuron being studied. Consequently, the eccentricity and the rotation of the array as a whole changed slightly from neuron to neuron but the overall display remained consistent. A standard set of colors based on the individual RGB guns of the color monitor, or equal additions of any two guns, was used. These colors were adjusted to an average value of $34 \mathrm{~cd} / \mathrm{m}^{2}$ by comparisons of the framc-avcraged values obtained from integrating the analog output of a EG\&G model 450 photometer fitted with a photometric filter. No attempt was made to select isoluminant colors for individual animals or neurons. Consequently, although it is considered unlikely, it is possible that luminance differences alone could account for the effects observed and therefore the use of the term "color" should be viewed as descriptive.

Each animal viewed the display monitor binocularly through an infrared reflecting mirror placed directly in front of its eyes. Eye position of the left eye was recorded with an infrared corneal reflection oculometer (Motter and Poggio, 1984). Computer software monitored eye position and immediately terminated any trial in which eye position exceeded a $1^{\circ}$ window centered on the fixation spot. The behavioral key and push buttons were located in front of the monkey at waist and midchest levels and were blocked from view by a partition. The monkeys worked daily for about 1200-1400 trials at a performance level of about $85 \%$ correct. Correct responses were rewarded after each trial. Animals were maintained on a controlled liquid intake schedule and received their baseline amount each day either in the course of working on the 

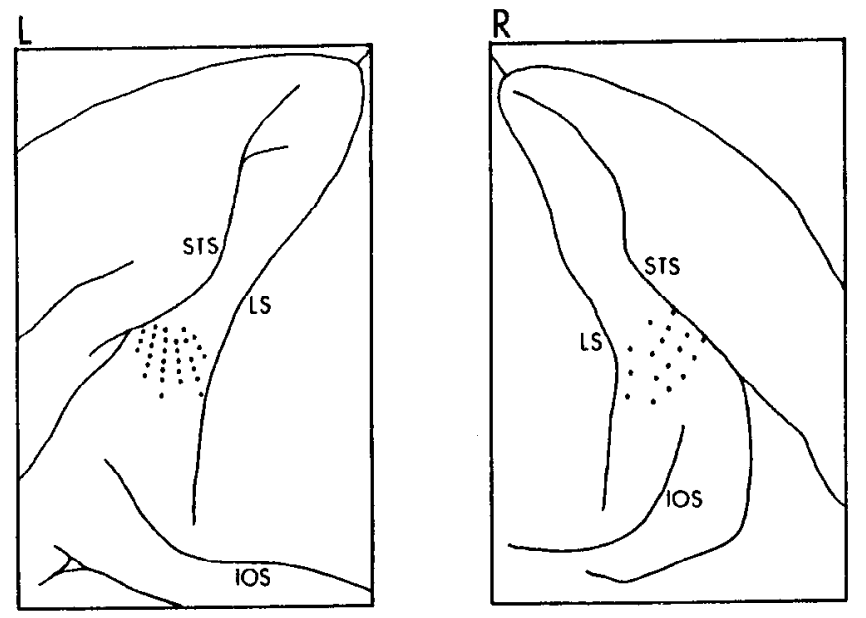

Figure 2. Locations of the penetrations into V4 in two of the four hemispheres studied. Line drawings were made from photographs. A reference grid was positioned to align with the histological guide pins, and the dots marking the locations of penetrations were placed with respect to the coordinates of the reference grid during the experiments. $L S$, lunate sulcus; $S T S$, superior temporal sulcus; $I O S$, inferior occipital sulcus.

tasks or supplemented afterward. Animals received weekly holidays from the schedule.

Data analysis. The spike rate activity was measured within defined periods of time during each trial. Averages of activity for each condition and for each neuron were combined in a repeated-measures analysis of variance (RM-ANOVA) design. Comparisons between specific conditions were obtained using a Student-Neuman-Keuls (SNK) multiple comparison procedure. Student $t$ tests were used during the experiments and in the final analyses to identify the presence of effects within individual neurons. Several indexes of activity differences are employed in the presentation of the data using the standard contrast index method that divides the difference between conditions by the sum of the conditions: $(A-B) /(A+B)$. Background activity was not subtracted from any of the response rates in this report.

Procedure. Single neurons were initially selected for study if they responded well to flashed stimulus bars of various colors and luminances that were about $2.5^{\circ} \times 0.5^{\circ} \mathrm{in}$ size. After quickly estimating the response properties, the receptive fields were mapped with flashed stimuli. The orientation and color/luminance preferences were then determined. Small adjustments of stimulus size were occasionally made to obtain optimal responses. From these results two orientations and two colors/luminances were chosen to form four possible stimuli. For neurons with clear orientation preferences the preferred orientation and the orthogonal nonpreferred orientation were chosen; the $45^{\circ}$ oblique orientations were used when no clear orientation preference was present. For neurons with clear color preferences, the two "best" colors or the best color and the best gray scale luminance were chosen. For neurons without clear color/luminance preferences, either a gray scale luminance pair or a combined color/luminance difference pair was chosen; often the latter was a color paired with black. The primary stimulus, composed of the preferred orientation and best color, was then used to redefine the edge of the receptive field nearest the fixation point and to examine the borders of the receptive field along an arc equidistant from the fixation target and passing through the receptive field center. Based on this mapping the spacing of stimuli in the array of the conditional discrimination paradigm was then determined so that only one stimulus was presented in the receptive field on any trial.

After all stimulus parameters were defined, the monkey was switched to the conditional discrimination paradigm described in Figure 1 . The final two bar stimuli were chosen according to a random permutation sequence for color, orientation, and location in the array. The receptive ficld stimulus was also chosen from a permutation sequence, although this sequence was often actively biased during the experiment against stimulus parameters that were ineffective in driving the neuron. It is important to note that the receptive field stimulus was not necessarily one of the final two stimuli presented; that is, there was no behavioral condition that was related to the receptive field position or the stimulus in it. Thus, only by chance was the receptive field stimuli one of the final two stimuli. After an initial study using the paradigm in Figure 1, other control paradigms were tested.

Three animals were trained and prepared for behavioral neurophysiological recording. Standard electrophysiological techniques were used to obtain recordings from cortical area V4 during the course of several months (Motter and Mountcastle, 1981; Motter, 1993). The impulse activity of single cortical neurons was recorded with glass-coated Elgiloy microclcctrodes inserted transdurally in to the cortex. Because recordings were to be made over several months and thus reconstruction of individual electrode penetrations would not be possible directly from histological sections, care was taken to define the extent of the prelunate gyrus in each hemisphere by a progression of penetrations from $\mathrm{V} 1$ across the lunate sulcus and prelunate gyrus and into the posterior bank of the superior temporal sulcus. Histological examination was used to confirm the neurophysiological localizations. After the last recording session the animals were deeply anesthetized with pentobarbital, the dura was opened within the recording chamber, and reference pins were inserted into the cortex using the positioning devices that set the coordinate grid for the microelectrode recording. The animal was then given a lethal overdose of pentobarbital and perfused with saline followed by buffered formalin. Photographs of the reference pins and brain were made through the recording chamber, and the brain was removed and prepared for histology. Reconstruction of the penetrations was based primarily on the observed physiology and construction of topographic maps using receptive field locations referenced to the grid defined by the reference pins in the photos. All experimental protocols were approved by the Animal Care and Use Committees at the VA Medical Center and SUNY-HSC.

\section{Results}

In each hemisphere the visual field representation of the lower contralateral quadrant cxtending out to $10^{\circ}$ was located on the crown of the prelunate gyrus. The location of the penetrations in two of the four hemispheres investigated is shown in Figure 2. Within area V4, 188 neurons with receptive fields centered $3-6^{\circ}$ from the fovea were fully studied with the simple discrimination (SD) paradigm. Neurons with receptive fields that did not extend to the fixation point were selected and of those 109 neurons were studied using the CD paradigm. Based on physiological signatures of cellular boundaries and on the measures of electrode position, neurons were sampled from both superficial and deep layers of V4. No correlations were observed between the cell depth and the physiological observations reported below.

The stimulus feature preferences of the V4 neurons were determined during an initial characterization of each neuron using $200 \mathrm{msec}$ flashed stimuli presented one at a time in the SD paradigm (Fig. 1). Although the examinations wcre nccessarily brief, the neuronal responses generally agreed with previous reports (Desimone and Schein, 1987; Haenny and Schiller, 1988; Schein and Desimone, 1990). Sixty-nine percent of the neurons displayed clear color preferences [(best - worst)/(best + worst) $>0.33$ ] among the six colors tested. The colors were photometrically equated for luminance and to the human observer appeared closely matched in brightness. Most of the V4 neurons also responded to white light, as has been previously noted (Schein and Desimone, 1990). The response to luminanceequated white stimuli averaged $70 \%$ of the response to the "best" color stimulus. Most neurons also responded to black stimuli, with population average response to black stimuli being about $90 \%$ of the response to the best color stimulus. The representations of black and white responsiveness could have been selectively biased in the population studied as the result of the routine use of black and white stimuli as the initial search stimuli during most electrode penetrations. 


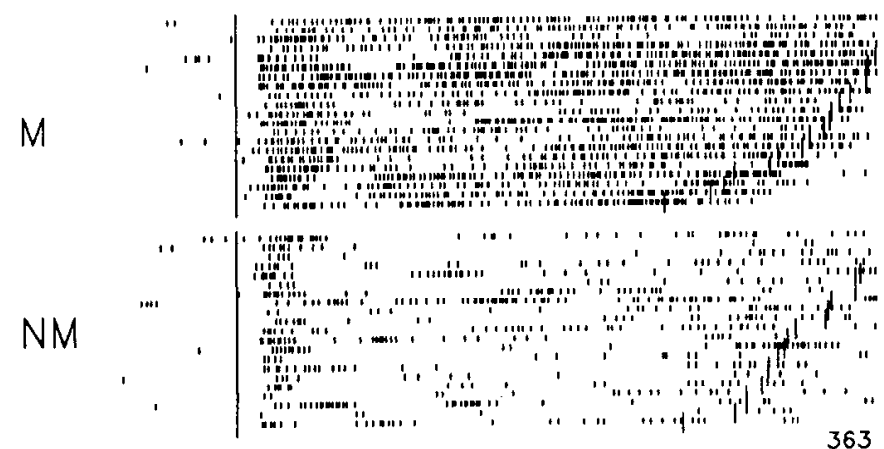

363

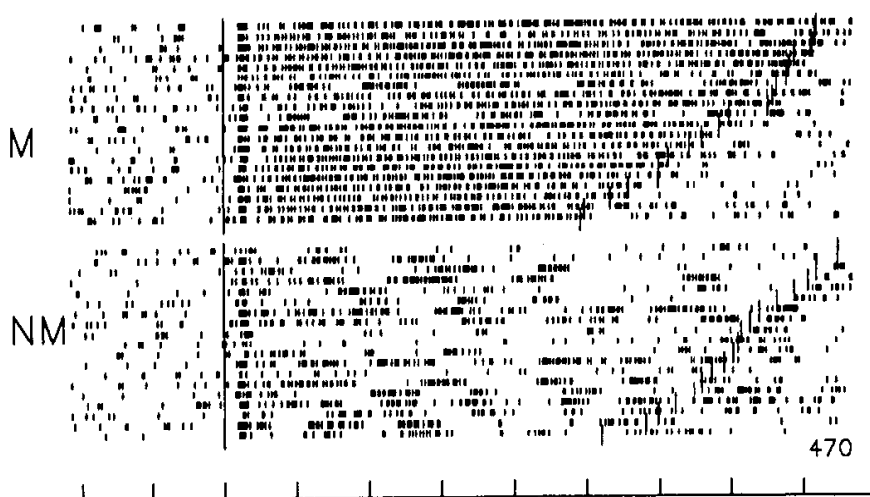

M
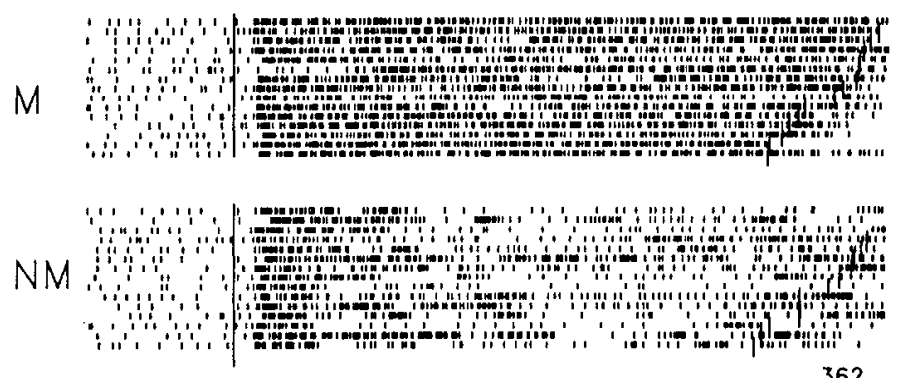

362

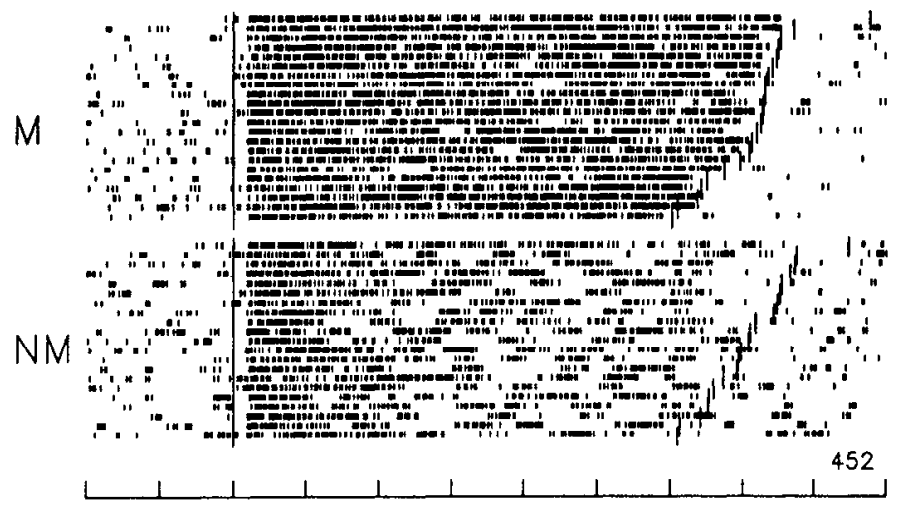

$300 \mathrm{msec} / \mathrm{div}$

Figure 3. Responses of V4 neurons in the CD paradigm. The raster displays show the differences between match $(M)$ and nonmatch $(N M)$ conditions for four V4 neurons. Each horizontal row represents a portion of a single behavioral trial synchronized in time to the onset of the array of stimuli indicated by the long vertical bars on the left. The short vertical tick marks represent the times of neural discharges. For each neuron the trials were run in an interleaved fashion and are sorted here according to stimulus conditions and trial length. The short staggered vertical bars along the right edge mark the time of release of the behavioral key near the end of the trial. The upper raster of each pair depicts the neuron's response to a stimulus when it matches $(M)$ the fixation spot color. The lower raster in each pair is the response to the identical stimulus when it does not match $(N M)$ the fixation spot color. Only the responses to the optimal stimulus (color and orientation) are shown; the responses to the other stimulus combinations that were interleaved in the experiment are not shown.

\section{Responses in the $C D$ paradigm}

A large majority ( 81 of 109) of the V4 neurons were differentially activated in the CD paradigm, as identified by individual $t$ tests $(p<0.05)$. All 81 neurons displayed a maintained activation when there was a match between the color/luminance of the receptive field stimulus and the color/luminance of the fixation spot. The same neurons displayed only a phasic activation if the color of the bar stimulus in the receptive field did not match the fixation spot color. The reverse was never observed; that is, match was always associated with activation, nonmatch with inactivation, or suppression. These observations were made for each of the four stimuli used in the CD paradigm. Each of the four stimuli, made from combinations of two colors and two orientations, usually elicited different responses with the predominant response difference occurring between stimuli of different orientations. The presentation of the results will be simplified by presenting only data for the two stimuli having the preferred orientation but different colors. Furthermore, the stimulus with the optimal color or luminance will be referred to as the primary stimulus, whereas the other stimulus of the pair having the same orientation will be referred to as the secondary stimulus.

Figure 3 illustrates the range of the differential effect. The responses to the primary stimulus for the matching (M) and nonmatching (NM) conditions are shown for four V4 neurons.
The trials displayed in each raster have been sorted according to the presentation duration of the array that varied from trial to trial. The trials are synchronized to the onset of the array indicated by the vertical bar on the left. The short, staggered set of vertical bars on the right mark the time of the release of the key by the monkey after the array had been reduced to two stimuli for $200 \mathrm{msec}$ and then blanked. In each case the $M$ condition results in a strong maintained activation and the NM condition results in a substantially reduced response. These differences were observed for pairings of different colors or grays of different luminances and for pairs of mixed color and luminance, studied in approximately three equal groups across the population. When the receptive field stimulus matched the conditional cue, and was therefore a potential discriminative target, the neurons responded with a prolonged discharge until the discriminative target was revcaled. On the other hand, when the receptive field stimulus did not match the cue and was therefore not a candidate for the final conditional discrimination, the neural activity was abbreviated.

The illustrations in Figure 3 are for the primary stimuli in each case, but the response to the secondary stimulus (same orientation, opposite color/luminance) also displayed the same effect, if that stimulus produced a response in the neuron. Figure 4 illustrates the responses to both sets of stimuli for one V4 neuron. This neuron had a strong color and luminance preference for blue and an equally strong preference for dark stimuli. 


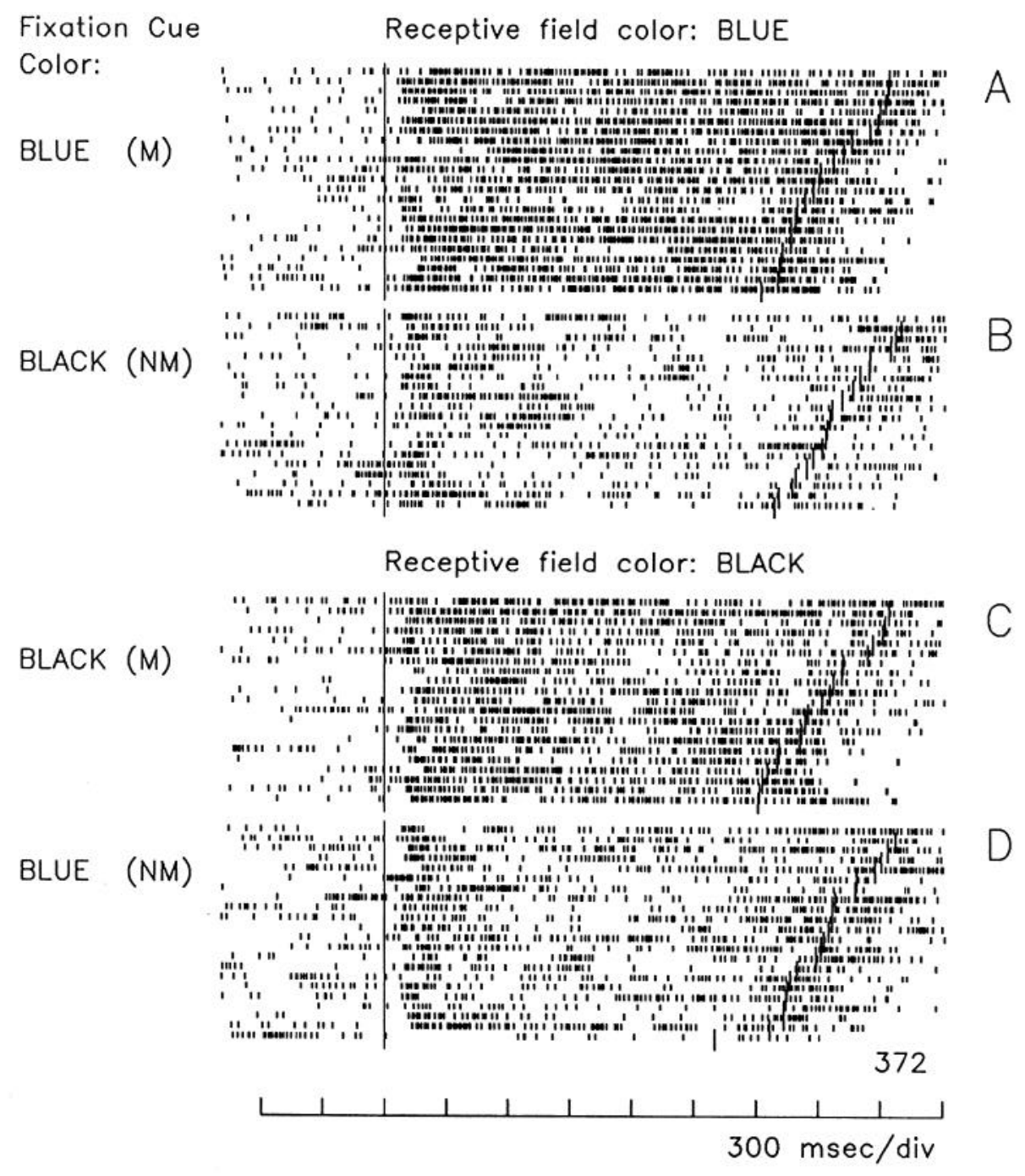

Figure 4. Responses to both primary and secondary stimuli in one V4 neuron during the $\mathrm{CD}$ paradigm. Presentation format is as in Figure 3. This neuron was responsive to both blue and black, but in the CD paradigm the responses were expressed differentially depending on the $\mathrm{M}$ condition.

Responses to both a blue (Fig. $4 A, B$ ) and a black (Fig. $4 C, D$ ) receptive field stimulus are shown for both conditions. Only the responses to the preferred orientation $\left(70^{\circ}\right)$ are shown. In Figure $4 A$ both the fixation spot color and the stimulus in the receptive field were blue. In Figure $4 B$ the fixation spot was black and did not match the blue stimulus in the receptive field. In the presence of a blue fixation spot, this neuron was strongly activated if a blue stimulus was within its receptive field (Fig. $4 A$ ). Similarly, this neuron was also activated in the presence of a black fixation spot when a black stimulus was within its receptive field (Fig. $4 C$ ). The neuron was markedly less active when the color of the stimulus in its receptive field did not match the color of the fixation spot (Fig. $4 B, D$ ). It is clear that under these conditions the activity of the neuron is not specific for a particular color or luminance, per se, but for stimuli with a selected color or luminance. It appears that the neuron is not "coding" for color or luminance but rather for a selection based on color or luminance.

\section{Population measures}

The mean discharge rate for each of the $109 \mathrm{~V} 4$ neurons during the last second of the array presentation was calculated for each condition. Figure 5 summarizes the results by plotting the match and nonmatch rates for the responses to the primary stimulus for each neuron. Differences as a function of match condition for the population studied were significant for both the primary and secondary stimuli (RM-ANOVA, $P<0.001)$. To establish the prevalence of the differences in the population, the responses of each neuron were examined with individual $t$ tests. By this measure 81 of the 109 neurons showed significant differences $(p<0.05)$. For purposes of display clarity the 81 neurons were broken into two groups and plotted on the left and middle in Figure 5 . The remaining 28 neurons not showing a clear difference between the matching conditions are plotted separately on the right in Figure 5. The difference between the activation in the match condition and the attenuated responses in the nonmatch condition are clear in most neurons. No examples were encountered in which the response to an NM condition was significantly greater than the response to the $\mathrm{M}$ condition. An estimate of the magnitude of the differential effect within the population of $\mathrm{V} 4$ neurons was obtained by calculating the ratio of the firing rates in the match condition over the rates in the nonmatch condition for each neuron. The mean of the distribution of these ratios is 2.3 and the median is 1.7. Thus, the activity associated with the primary stimulus in the match condition is about twice the activity elicited by the same stimulus in the nonmatch condition.

Both the primary and the secondary stimulus produced com- 


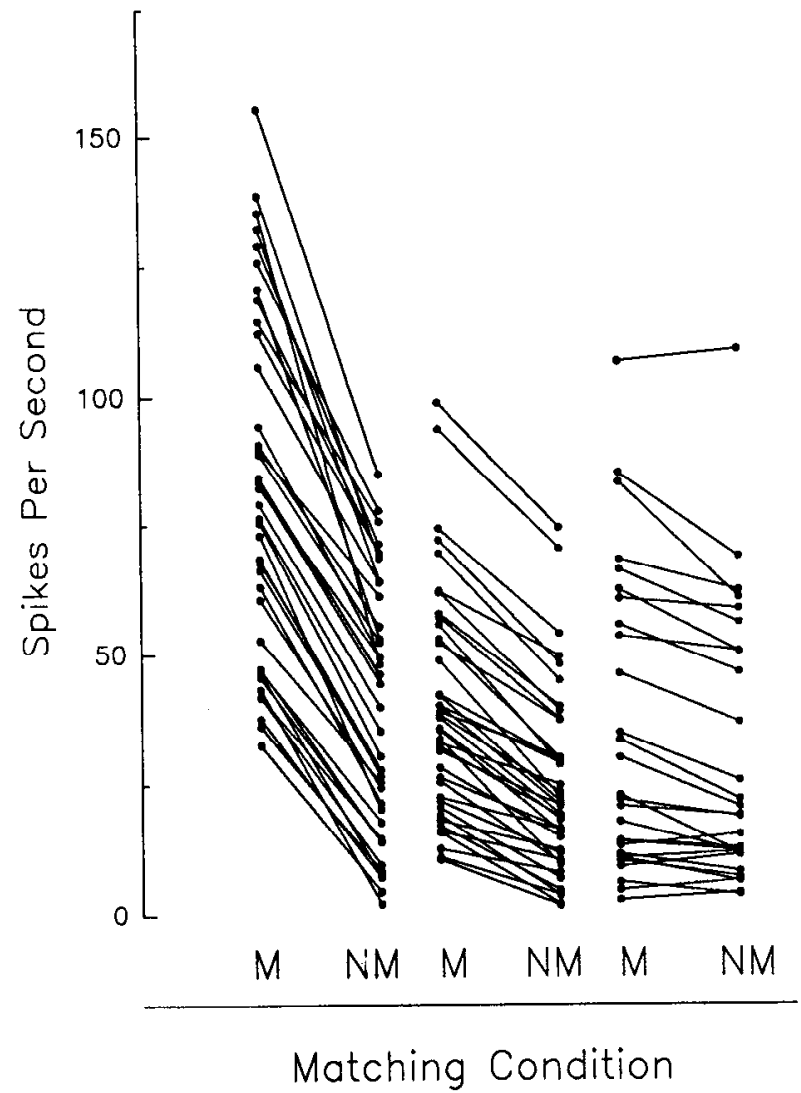

Figure 5. Mean discharge rates for match $(M)$ and nonmatch $(N M)$ conditions for the primary stimulus for each of the 109 neurons. The 81 neurons having significant differences between conditions were ranked by difference between conditions and divided by rank into two groups, plotted here on the left and in the middle. The data for the remaining 28 neurons are plotted on the right. The average firing rate for each condition is joined by a line for each neuron. Discharge rates in match conditions exceeded those in nonmatch conditions for each significantly different case.

parable outcomes in the $M$ conditions. The match versus NM comparison was significantly different for both sets of stimuli (RM-ANOVA, SNK comparisons, $P<0.05$ ). Comparisons for individual neurons were made for the 96 neurons that had detectable responses to the secondary stimulus $(p<0.05, t$ test, response vs baseline). A response index comparing the $M$ conditions for each stimulus was calculated using the response rates from the final $1 \mathrm{sec}$ of the array presentation. A standard contrast index of the form $(M-N M) /(M+N M)$ was used, where $M$ is the response in the match condition and $N M$ is the response in the nonmatch condition. Figure 6 shows a scatter histogram of these indexes for the primary and secondary stimuli. The average index was 0.29 for the primary stimulus and 0.22 for the secondary stimulus. As shown in Figure 6, the differential activation that occurs during the trial is based on whether or not the receptive field stimulus matches the conditional cue.

\section{Stimulus-stimulus interactions}

With multiple stimuli in the visual field one of the obvious considerations is whether the observed effects could be explained by interactions between stimuli either within the classical receptive field or between the classical receptive field and the suppressive surrounds that have been observed in V4. In-

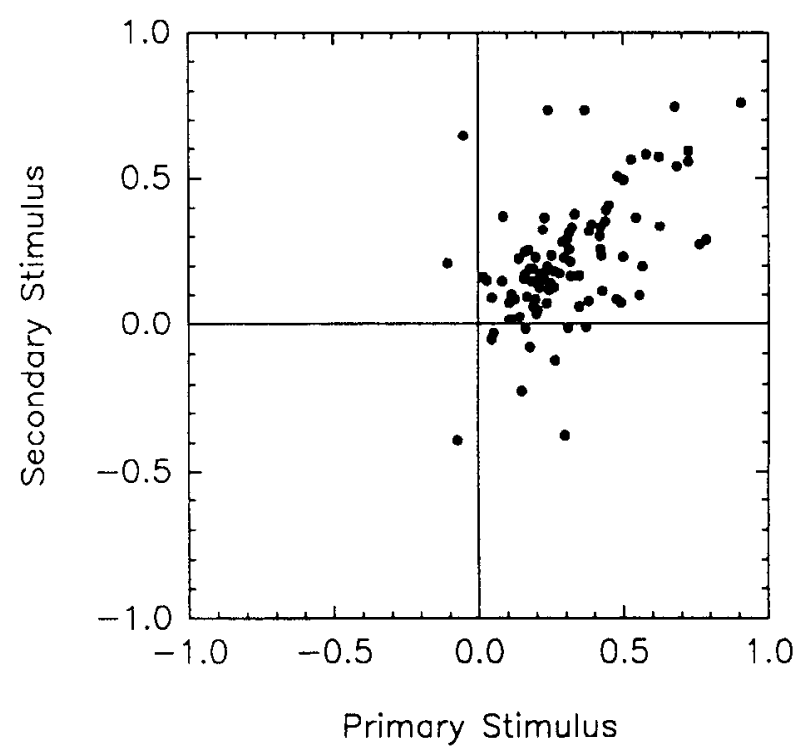

Figure 6. Distribution of the contrast indexes for the responses to primary and secondary stimuli during the $C D$ paradigm. Positive contrast values indicate that the match condition produced a larger response than the nonmatch condition. Each data point represents one neuron.

teractions between the array stimuli in the CD paradigm can be ruled out because the same stimulus sets could be observed to result in different responses depending on the color of the fixation spot. The array stimuli were randomly chosen on a trialby-trial basis and therefore the number of instances of exact replication of the arrays was fairly small in the standard CD paradigm. However, sufficient comparisons were available between any two stimulus locations to rule out simple interactions. Furthermore, the cue switching paradigm presented in the following companion article (Motter, 1994) provides a complete control for interactions between array stimuli, as well as presenting further experiments examining the relationship between stimulus positions in the array and the classical receptive field.

Although the fixation spot was carefully determined to lie outside the classical receptive field of the neurons studied, the possibility remained that the fixation spot was in a subthreshold area or located in the suppressive surround area. This possibility was examined using the SD task shown in Figure 1 (right) to present the same color or luminance combinations under different conditions. For 66 of the 109 neurons a variation of the SD paradigm was used to present the stimulus combinations for a duration of $2 \mathrm{sec}$ in order to match the duration of the CD task. The SD task provided the same physical stimulus relationships between the fixation spot and the receptive field stimulus, but the viewing conditions did not require the subject to process actively information about the peripheral stimulus. The SD task required the monkey to ignore peripheral stimuli while fixating a central spot and to make an orientation discrimination of a small bar flashed directly over the fixation spot irrespective of the color or luminance of the fixation spot or of the bar flashed over the fixation spot. Thus, the animal was required to perform the same type of task, an orientation discrimination, but was required only to attend to the location of the fixation spot.

Under these conditions two general classes of responses were observed for V4 neurons. Neurons displayed either a maintained or a phasic response during the 2 sec stimulus period. Examples are shown in Figure 7 for two neurons whose responses in the 


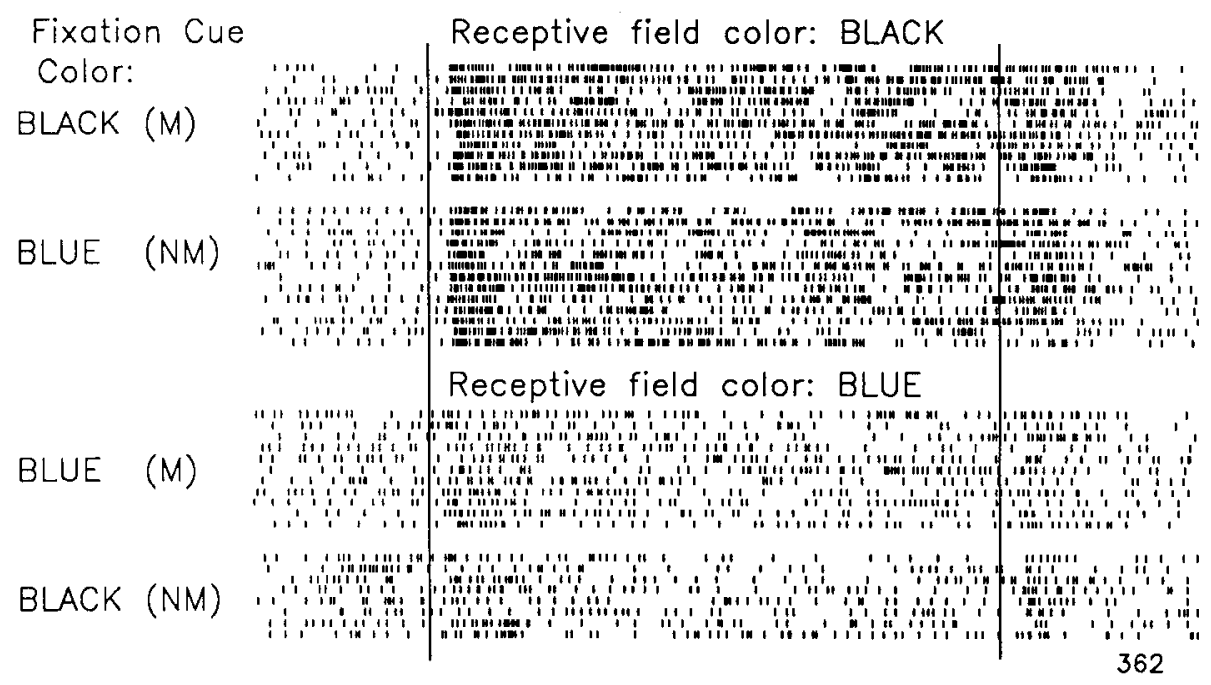

Figure 7. Responses in the SD paradigm to the same color combinations used in the $C D$ paradigm. In the SD paradigm there was no required behavioral response based on color or luminance, and only the fixation spot and receptive field stimulus were presented. Onset and offset of the $2.0 \mathrm{sec}$ stimulus period are marked by the vertical bars on the left and right. In contrast to the $\mathrm{CD}$ paradigm, the responses in the $\mathrm{SD}$ paradigm are dependent only upon the receptive field stimulus.

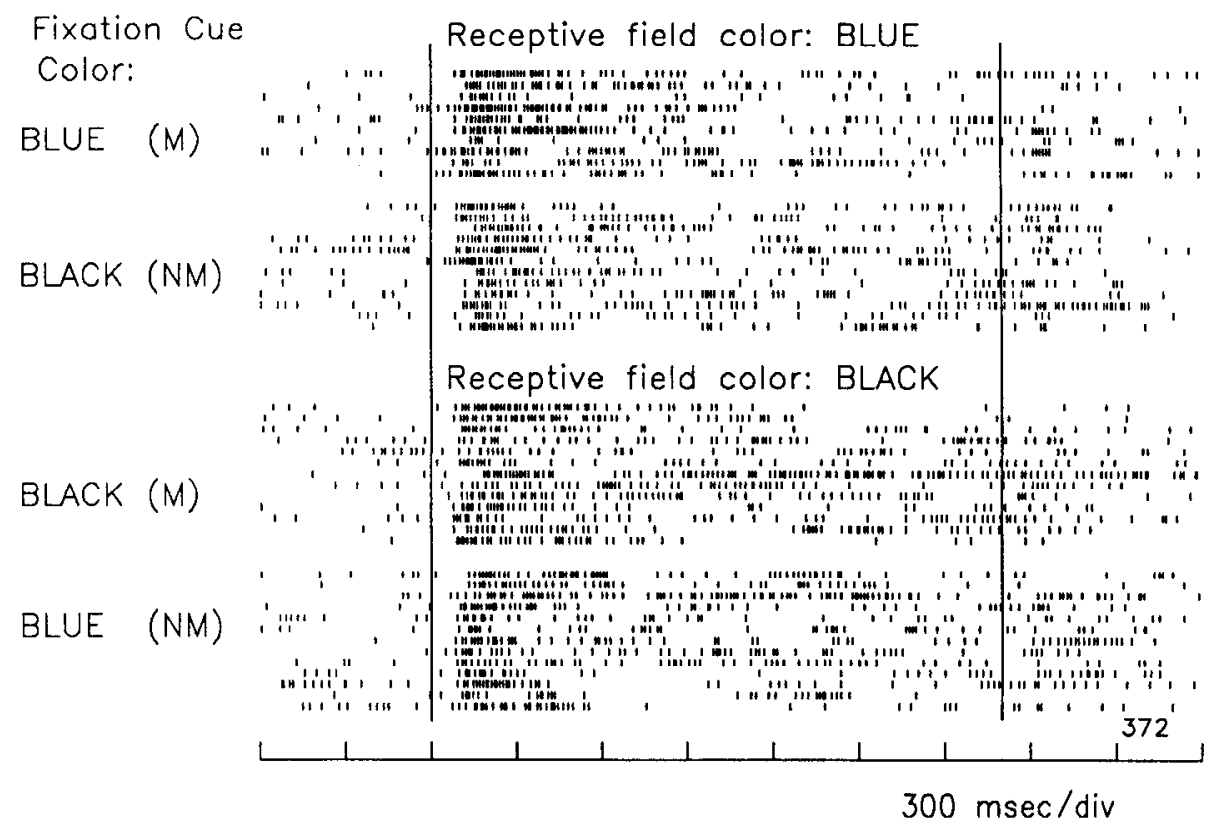

CD paradigms are shown in Figures 3 and 4 . Both of these neurons were studied with a black and blue stimulus combination. For each of the four pairs of rasters the upper part is the response during a match $(\mathrm{M})$ condition where the fixation spot and the receptive field stimulus have the same color. The lower raster of each of the four pairs shows the response when there is a nonmatch (NM) between fixation spot and stimulus color. In each case the responses during this simple task appear to be dependent upon the stimulus in the receptive field and show little correlation to the color of the fixation spot. The response rate during the last sccond of the stimulus period was measured for M and NM conditions of the primary stimulus in a subset of 66 of the neurons fully studied in the CD paradigm. The $\mathrm{M}$ and $\mathrm{NM}$ conditions for the $\mathrm{SD}$ were not significantly different ( $P>0.05$, RM-ANOVA SNK comparisons). $T$ tests again were used to identify the effects in individual neurons. For 60 of the 66 neurons the simple pairings of central and peripheral stimuli were not able to reproduce the selection effect; instead the responses were determined by the receptive field stimulus alone. Six of the 66 neurons had small but significant differences ( $t$ test, $p<0.05$ ) between $\mathrm{M}$ and NM conditions of the SD task; however, only three of these six neurons displayed significant differences in the CD paradigm. These results indicate that the feature-selective effect seen in the CD paradigm is not a simple interaction of central and peripheral stimulus colors.

\section{Activation versus suppression}

The differences in discharge activity during the $\mathrm{CD}$ paradigm result from both activation and suppression relative to the discharge during the SD paradigm. Activation during the match condition can be clcarly secn in ncurons with normally phasic responses that then develop a maintained activation like that shown for the neuron (372) in Figures 4 and 7. Suppression or inactivation can be clearly seen for neurons with normally maintained activity that is depressed during the nonmatch condition as shown for the neuron (362) in Figures 3 and 7. To quantify these observations a contrast index was calculated for match and nonmatch conditions comparing the response in the $\mathrm{CD}$ paradigm with the response in the SD paradigm. These data were obtained for the same 66 neurons studied with 2 sec stimulus periods in the SD paradigm described above. Response 
rates were based on activity in response to the primary stimulus during the last second of each condition. A contrast index value for each neuron was obtained for the match condition according to $(M-B) /(M+B)$ where $M$ is the response in the match condition of the $\mathrm{CD}$ paradigm and $\mathrm{B}$ is the simple average of the responses to match and nonmatch conditions in the SD paradigm. Because the latter responses were not significantly different, the average response was used to establish a single baseline for the comparisons. A contrast index value for the nonmatch condition was obtained in a similar manner: (NM B) $/(\mathrm{NM}+\mathrm{B})$, where $\mathrm{NM}$ is the response in the nonmatch condition of the $C D$ paradigm. The resulting indexes for each neuron are plotted in Figure 8. Positive values for the match condition indicate an activation in the $C D$ paradigm relative to the "baseline" response in the SD paradigm. Negative values for the nonmatch condition indicate a suppression during the CD paradigm relative to the response in the SD paradigm. The data points for those neurons that had significant differences between conditions in the CD paradigm are shown as solid circles; those that did not are shown as open circles. Many neurons, those in the upper left quadrant, show both activation during match and suppression during nonmatch conditions in the CD paradigm. For a few neurons the differences in the $C D$ paradigm apparently reflect a relative difference in an overall pattern of response activation or suppression. The few cases that were below the diagonal where the response to an NM condition was greater than the response to the $\mathbf{M}$ condition were not significantly different.

\section{Temporal development of the differential response}

It has been demonstrated that cuing information from nonvisual sources can influence V4 neuronal activity prior to the presentation of visual stimuli (Haenny et al., 1988; Maunsell et al., 1991). Therefore, the data were examined for evidence that neural activity was biased by the cue color/luminance itself in the interval before the array was presented. In the present experiments the conditional color cue, the fixation spot, was available from the start of the trial for a period greater than $0.5 \mathrm{sec}$ before the peripheral stimuli were presented. The activity in this prearray period was grouped according to the conditional cue and differences tested on an individual cell basis. None of the 109 cells showed a significant difference in activity related to cue color or luminance during this period ( $t$ test, $p<0.05$ ). Thus, the data provide no indication that the discharge activity of the neurons was sensitive to the cuing conditions prior to the presentation of the stimulus arrays.

The time course of the development of the differential response during the array presentation was examined for the population of $109 \mathrm{~V} 4$ cells. First the average discharge rate for each neuron was determined in sequential $10 \mathrm{msec}$ intervals during the presentation of the array for each separate condition. Then the response rate for each neuron was normalized with respect to the interval with the highest response rate across all conditions. The normalized data from different neurons were then averaged for each $10 \mathrm{msec}$ interval and for each stimulus condition. Figure $9 A$ plots the results obtained for the primary stimulus of each color/luminance pair. The upper line is the average response of the 109 neurons during the match condition; the lower line, the average response during the nonmatch condition. The population latency to the presentation of the array can be seen to be about $50 \mathrm{msec}$. Although differences between conditions could be seen to occur in individual neurons at the

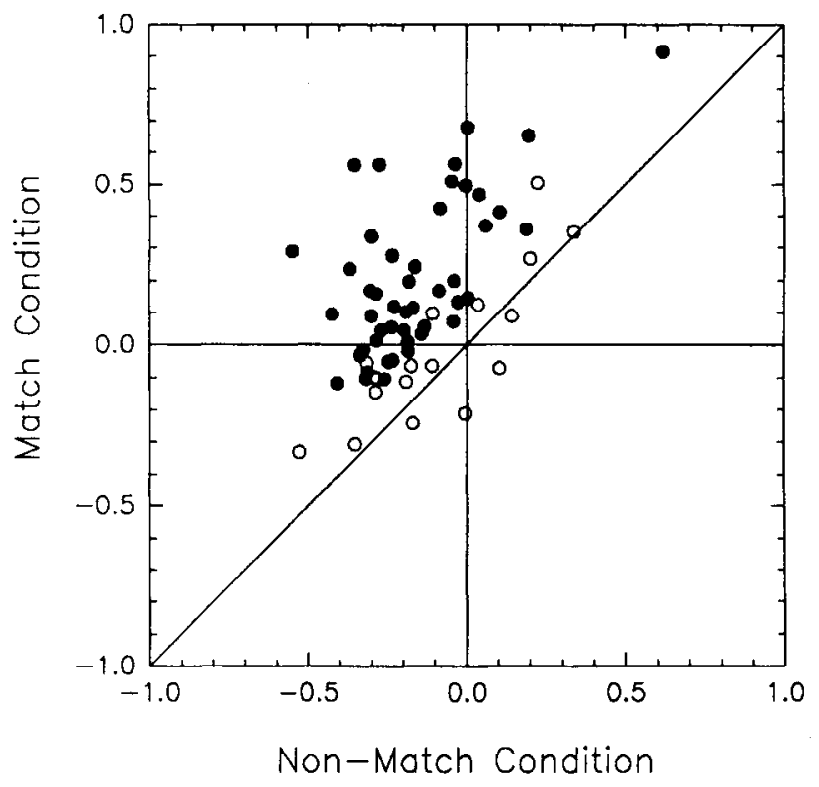

Figure 8. Comparison of activation and suppression in CD paradigm Each data point compares the response in the $C D$ paradigm with the response in the baseline SD paradigm for both $\mathrm{M}$ and NM conditions. Values are expressed in terms of a contrast index, with positive values indicating activation and negative values indicating suppression relative to the SD task. Solid circles represent neurons with significant differences between $\mathrm{M}$ and $\mathrm{NM}$ conditions in the CD task; open circles represent neurons showing no differences between those conditions.

onset of the response (see Fig. 3), for the population response both conditions result in comparable initial activations. The differential activation develops over the first few hundred milliseconds of the response. Because the comparison in Figure $9 \mathrm{~A}$ was created from the responses to stimuli optimized for each neuron, it should represent an optimal temporal response profile for the emergence of the feature-selective effect in area V4. The responses to the secondary stimulus of each color or luminance pair, on the other hand, might provide a more general profile because they represent the response to less than optimal stimuli. The temporal profiles of the responses to secondary stimuli are plotted in Figure $9 B$. The profiles of temporal development of the differential responses were similar with both stimuli. Differentiation between match and nonmatch conditions begins to occur about 150-200 msec after the array is presented. Although the population response declines after the initial appearance of the stimulus array, the difference between match and nonmatch conditions gradually increases during the first $500 \mathrm{msec}$ and then remains relatively constant for the remainder of the trial.

The data in Figure 9 model the time course of the net activation of area $\mathrm{V} 4$ neurons during the conditional discrimination trial. The data show that after about $150-200 \mathrm{msec}$ the stimuli that arc potential targets for a discriminative task begin to be represented by a greater level of activity in the population than other stimuli. After 400-500 msec the stimuli that are potential targets are evoking about twice the level of activity above background as the nontargets.

\section{Stimulus feature preferences}

Quantitative examination of stimulus preferences were not attempted in the CD paradigm in this series of experiments, but qualitative comparisons could be made between the responses to the colors or luminances and the orientations used in the 

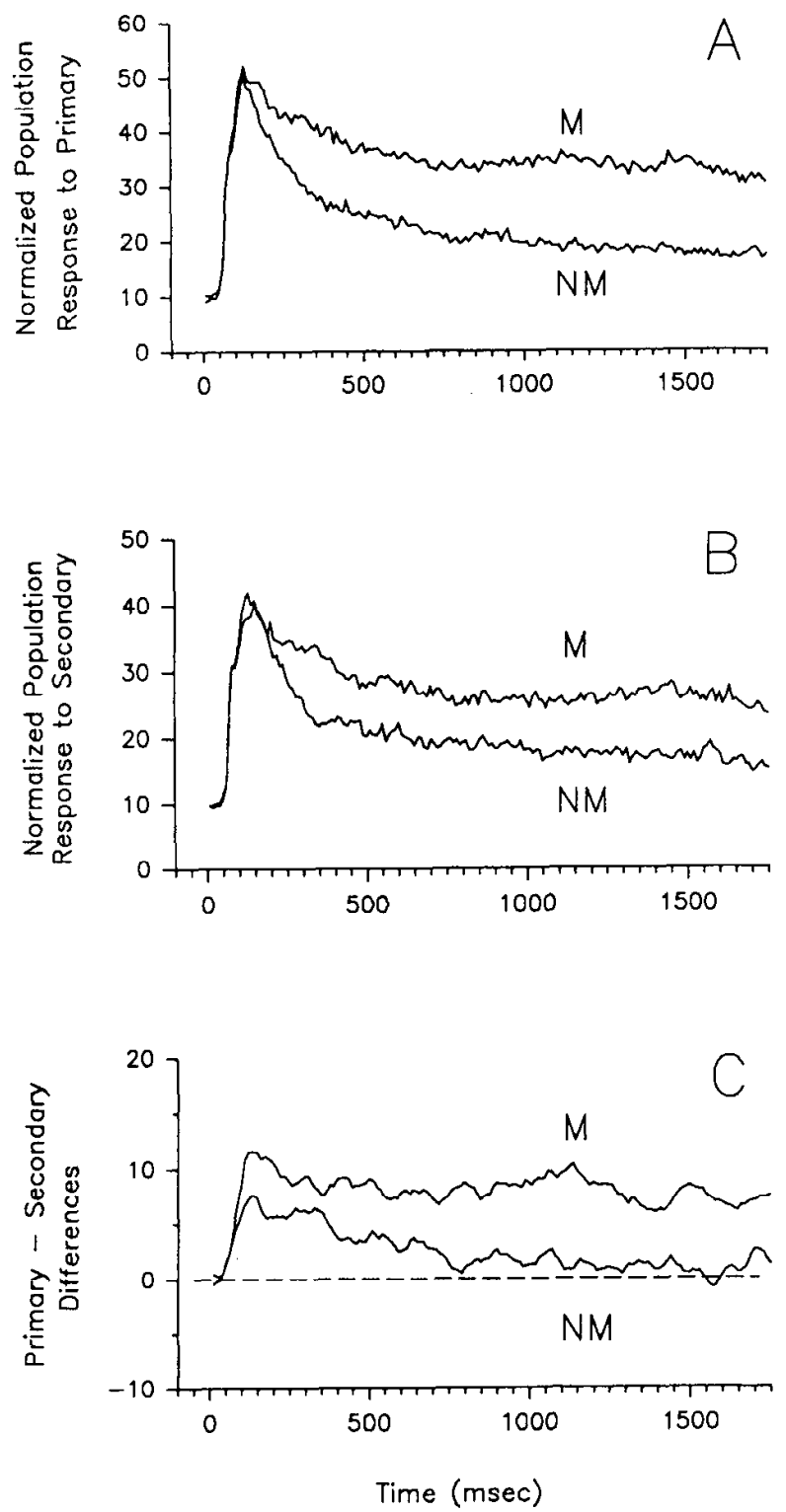

Figure 9. Temporal development of the differential response: normalized population histograms of the response to primary $(A)$ and secondary $(B)$ stimuli during match $(M)$ and nonmatch $(N M)$ conditions in the $C D$ paradigm. The differential response develops over the first few hundred milliseconds. The differences between the two $M$ conditions and the two NM conditions are shown in $C$. The response difference between primary and secondary stimuli persists throughout the trial for $\mathrm{M}$ but not for $\mathrm{NM}$ conditions. Histograms are based on 10 msec bins. For each neuron the bin with the greatest activity across all conditions was set to 100 and the remaining bins normalized to that value. Data were then averaged across the 109 neurons. The abscissa represents time synchronized to the onset of the stimulus array.

primary and secondary stimulus choices. Neurons that were selectively responsive to particular colors, luminances, or orientations in the SD paradigm remained sensitive to those same parameters in the $C D$ paradigm. This was clearly true for the match condition in the $C D$ paradigm, as can be judged from the difference between the population response to the primary and secondary stimuli in the match condition of Figure $9 C^{\circ}$. While it remains possible that quantitative measures could reveal shifts in preference between stimulus parameters that were judged to be near equivalent at the time of the experiment, it is clear that the basic response differences were maintained in the $C D$ paradigm. On the other hand, distinct sensitivity differences were more difficult to observe in the nonmatch conditions. This can be seen in the population response in Figure $9 C$, where differences in the response to the primary and secondary stimuli gradually disappeared during the trial. These distinctions were significant in that the RM-ANOVA SNK comparisons (SNK $p<0.05$ ) showed differences between all comparisons (color vs matching) except for the comparison between NM conditions. The relative lack of stimulus feature selectivity in the nonmatch level of activity suggests a possible distinction between the processes that control the mechanisms of activation and those that govern inactivation or suppression within V4 during the $C D$ paradigm. Within the $C D$ paradigm, the attenuation associated with the nonmatch condition occurred regardless of whether the neuron was sensitive to the fixation point color itself (when presented within the receptive field).

The relationship between color, luminance, or orientation sensitivity and the occurrence of a differential response in the CD paradigm was examined. Response contrast indexes for color, luminance, and orientation sensitivity were derived from the responses in the SD paradigm and were compared with the response indexes describing the matching condition of the $C D$ paradigm. For the color values the best and worst responses to the six colors tested were used. For the luminances the responses to the black and white stimuli were used. For the orientation values the responses to the preferred and orthogonal orientations were used. These values are plotted in Figure 10 against the contrast index calculated for the response to the primary stimulus in the CD paradigm for 100 of the neurons. No clear general relationship between color, luminance, or orientation sensitivity and the presence of the effect is evident. Exploratory multiple linear regressions using these indexes and others representing ratios of black:color and white:color responses did not reveal any meaningful predictive relationships. What is clear, however, is that the behavioral modulation attributable to the selective attentive effect is of a comparable magnitude to the level of control exerted by the stimulus characteristics themselves. The median index value for color was 0.43 , for luminance 0.21 , for orientation 0.25 , and for the matching effect 0.27 (the median for the 81 neurons with a significant difference between $M$ conditions was 0.32 ).

\section{Discussion}

The results of the present study indicate that a behavioral requirement for the selection of a stimulus with a particular feature, either color or luminance, can determine the responsiveness of V4 neurons to stimuli presented within their classical receptive field areas. The selection is based on the presence or absence of the stimulus feature and is independent of spatial location. The activity reflects a selection based on the cued feature and not simply the physical color or luminance of the receptive field stimulus. Across the population of V4 neurons, stimuli that match the selected color or luminance elicit about twice the activity as stimuli that do not match the selected color or luminance. These feature-selective changes in activity were observed to develop fully over a period of about $500 \mathrm{msec}$ and to be maintained over the remaining $1-2 \mathrm{sec}$ of the behavioral trial. These physiological observations suggest that stimuli that possess a unique color or luminance feature can be preferentially selected in parallel across the visual field. 


\section{Feature sensitivity versus feature selection}

The feature-specific modulatory processes demonstrated by Haenny and Schiller (1988) for V4 neurons were associated with the neuronal sensitivity to a particular stimulus dimension that was relevant to the completion of an alternating sequence task. When the animal was required to attend to changes in orientation or color, the respective sensitivities for those receptive field characteristics were sharpened; thus, increased responses were observed for preferred stimulus parameters and decreased responses were observed for nonpreferred stimulus parameters. The degree of feature-specific modulation was correlated with how sharply tuned the neuron was for that particular stimulus dimension. A sharpening of sensitivity to the appropriate stimulus dimension as a function of task difficulty was also reported by Spitzer et al. (1988) for V4 neurons. They demonstrated that task conditions that were more difficult and therefore demanded a higher level of attention were associated with higher rates of neural activity and narrower orientation bandwidths. In contrast, the feature-selective activity reported in this article appears to have little to do with either the task difficulty or the particular sensitivity to the stimulus dimension per se, but rather appears to be associated with the act of selection of stimuli having a particular stimulus feature.

In other studies Haenny et al. (1988) and Maunsell et al. (1991) reported that the responses of V4 neurons to visual stimuli in a delayed match-to-sample task could be modulated by either visual or somatosensory sample cues. Their task required the detection of a cued grating orientation in a scqucntial scrics of grating presentations. The interactions between the sample cue (delivered at the onset of the trial) and the visual receptive field stimuli in those studies were quite different than those reported here. They reported a wide mixture of results from various cue and stimulus combinations. Some V4 neurons responded best to any of the visual grating orientations whenever the sample cue had a specific orientation, while other V4 neurons responded best to particular but not necessarily matching combinations of cue and receptive field stimuli, and still others were selectively responsive to the sample cue information even before the visual stimuli were presented. Although there was no special relationship between $\mathbf{M}$ conditions and neural activity across the set of observations, their results clearly indicated that information provided by the cue sample did differentially affect the later neural processing of visual grating patterns. Maunsell et al. (1991) further demonstrated that the particular form of the modulation that occurs is not dependent upon the modality, either visual or somatosensory, of the cue.

The feature selectivity observed in the present report was quite different from that described in the above studies. Unlike the pairing of a particular cue orientation with a particular effect in any given neuron in the studies of Haenny et al. (1988) and Maunsell et al. (1991), the selectivity effect observed in the present study was generalized for each neuron. What was important was the match, not the particular color or luminance of the stimulus. Also differing from the above studies, the feature selectivity effect was always the same in the present study. The match condition determined the activity. A match between cue and receptive field stimulus was associated with activation, whereas a nonmatch was associated with an attenuated response. While the effects in the present study are more uniform, it is clear that the V4 studies have used quite different tasks to address the role of attention in information processing and have
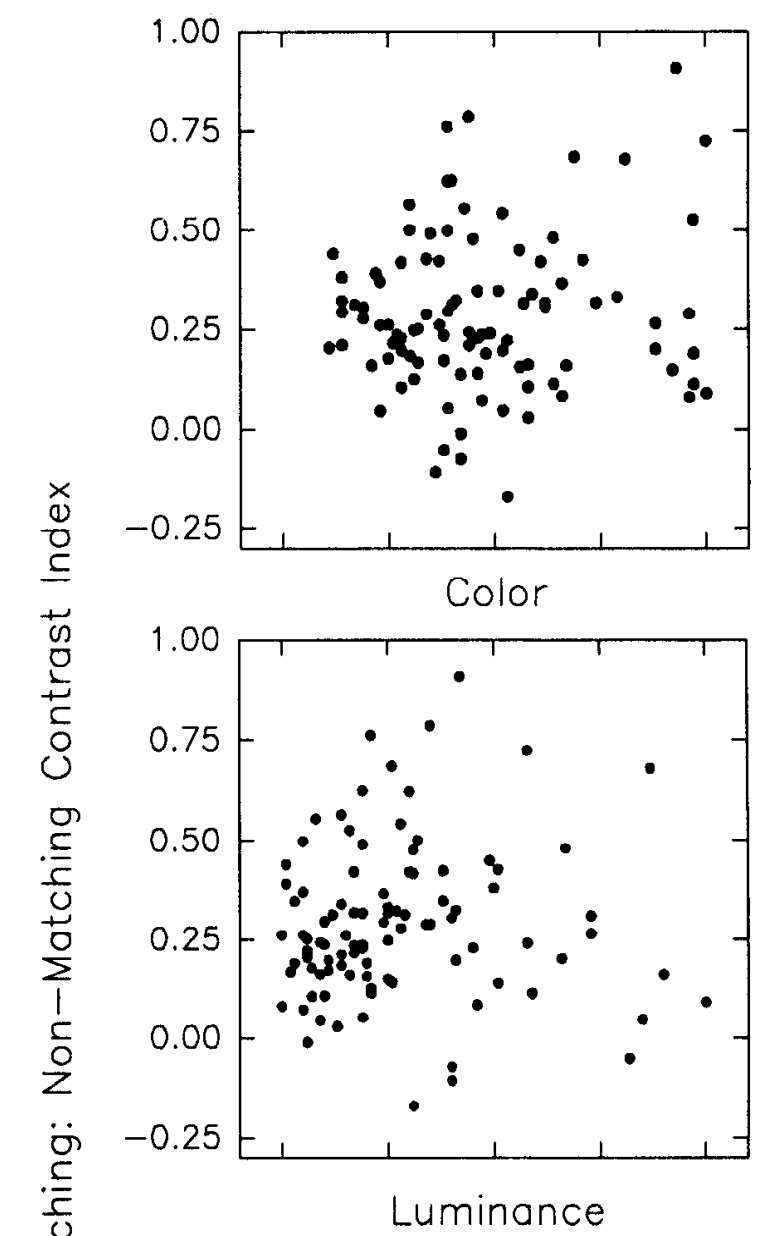

$+$

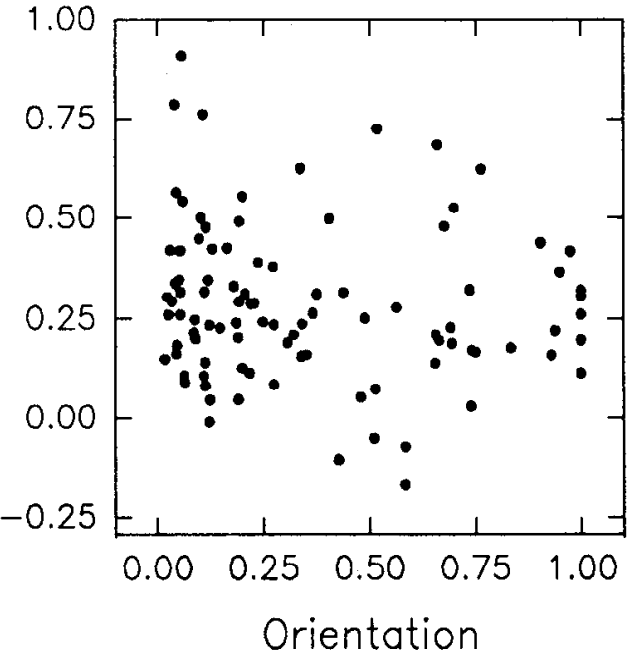

Figure 10. Relationships between color, luminance, and orientation sensitivity and the differential response associated with the color or luminance $\mathbf{M}$ condition. Color, luminance, and orientation sensitivity were assessed from the responses to $200 \mathrm{msec}$ flashes presented in the SD paradigm. The differential responses were measurcd over a 1 sec interval in the CD paradigm.

assessed the effects during quite different time frames within the tasks.

Moran and Desimone (1985) reported that spatially restricted focal attention suppresses responses to irrelevant stimuli within the receptive fields of $\mathrm{V} 4$ neurons when both relevant and ir- 
relevant stimuli are located within the receptive field. However, when only one of the two simultaneous stimuli was presented in the receptive field, either the relevant or the irrelevant stimulus, the responses were equivalent and not suppressed. From these data they formed the hypothesis that focal attention directed at a particular spatial location acts to filter out irrelevant stimuli at that location. Physiologically this was seen in terms of the suppression of responses to irrelevant stimuli that were present in the receptive fields of individual V4 neurons, but only when focal attention was directed to other (relevant) stimuli in the receptive field. Recently it has been shown that within the context of an array of competing stimuli, focal attention needs only to be directed to the receptive field location, not necessarily to a second stimulus in the receptive field (Motter, 1993). In contrast to the spatial selectivity seen with directed focal attention, the feature selectivity described in this article is not spatially selective. The stimulus feature match (color or luminance) occurs between a cue at the fixation point (or held in memory as reported in the accompanying article) and the stimulus in the receptive field. Both an activated response to matching stimuli (that are relevant because they are potential discriminative targets) and an attenuated response to nonmatching stimuli (irrelevant stimuli) are observed when either set is presented alone in the receptive field. The feature-selective responses are thought to occur in parallel across the visual field. This seems a reasonable conclusion given that the positions of the final two stimuli in the CD paradigm were not correlated with the receptive field location being studied and that the responses were consistent across trials. The data therefore are taken to indicate that all matching stimuli in the visual field result in neural activation in $\mathrm{V} 4$ at the appropriate topographic locations. The consequence of the selective process is that on any given trial the clear majority of the highly activated V4 neurons preferentially represent those separate stimuli in the visual scene that correspond to potential targets as defined by their match to the cue's color or luminance. A sequential combination of these two processes, initially a full-field prefocal attentive selection based on features followed by a spatially restricted focal attentive process (as described by Moran and Desimone, 1985), offers an interesting physiological model of selective attention expressed within single neurons in V4.

\section{Color sensitivity versus color selection}

Many neurons were sensitive to a restricted set of colors or luminances that were defined during the initial classification of the neurons in the SD paradigm. These sensitivities are retained during the match condition of the CD paradigm, as can be seen in the average population response. The results also clearly indicate that in the $C D$ paradigm the activity of the V4 neuron is not controlled by the presence of a particular stimulus wavelength or apparent color but instead the response is gated by the selection of a particular stimulus color or luminance. The data suggest that the range of colors or luminances to which a neuron can respond is limited by the sensitivities measured in the SD paradigm, but the response on any given trial in the CD task is gated by the conditional cue. Thus, on one trial a neuron may be highly activated by a white stimulus but poorly activated by black or red stimuli, on the next trial it might be activated by a black but not white or red stimuli, and on the next trial it might be activated by red but not black or white, all depending upon which color or luminance is cucd for that trial.

Previous physiological studies have emphasized the potential role V4 may play in the discrimination of color and form and the recognition of color constancies (Zeki, 1983; Schein and Desimone, 1990). While the emphasis of the present results and discussion is placed on the selection of items by color, it is recognized that the observations made in the $\mathrm{CD}$ paradigm are during periods of time before spatially directed focal attention is required. The change from one attentive state to the other results in major changes in the processing of visual information in V4 both in the area of directed attention and in peripheral areas away from the area of focal attention (Moran and Desimone, 1985; Mountcastle et al., 1987; Spitzer et al., 1988; Maunsell et al., 1991; Motter, 1993). It is therefore reasonable to expect that the role V4 plays in color perception may vary considerably with the processing requirements of the task.

The present data suggest that the major role area V4 plays in color vision may be the grouping (or separation) of objects based on color or luminance. Within the CD paradigm this appears to be nearly a bistable process resulting in a figure/ground-like representation of matching versus nonmatching stimuli across the population of $\mathrm{V} 4$ neurons. In other circumstances these processes may work over a more localized area with other features being emphasized in other locations. Interpreted in this manner, the results suggest that lesions of V4 may not result in straightforward deficits in color discrimination but may present deficits in tasks requiring higher-order types of comparisons or searching skills. The deficits resulting from lesions of $\mathrm{V} 4$ have in fact been quite varied in their detection of perceptual loss (Dean, 1979; Wild et al., 1985; Heywood and Cowey, 1987; Heywood et al., 1992) and have implied a more complex role for V4 than the discrimination of basic visual features (Schiller and Lee, 1991).

In summary, the attentive pressures imposed by the $\mathrm{CD}$ task used here create conditions for visual processing within area V4 that are not present under passive viewing conditions, as evidenced by a lack of response differentiation for matching conditions during the SD paradigm. This process appears to occur in parallel across the visual field and requires several hundred milliseconds to achieve the maximum separation of matching classifications. Thus, the observed feature-selective processing appears not to be automatic but rather an active condition, perhaps requiring feedback control. These observations indicate a process with properties that are somewhere between the passive preattentive grouping processes and the focal attentive, spatially restricted, serial processes described in psychophysical studies of visual search. The consequence of the selective pressure is that the population of V4 neurons that are highly activated represents the stimuli in the visual scene that correspond to potential targets. Under these conditions the scene is not segmented into many "color" maps, but into two maps, potential targets and background objects.

\section{References}

Bergen JR, Julesz B (1983) Parallel versus serial processing in rapid pattern discrimination. Nature 303:696-698.

Cavanagh P, Arguin M, Treisman A (1990) Effect of surface medium on visual search for orientation and size features. J Exp Psychol [Hum Percept] 16:479_491.

Corbetta M, Miezin FM, Dobmeyer S, Shulman GL, Petersen SE (1991) Selective and divided attention during visual discriminations of shape, color, and specd: functional anatomy by positron emission tomography. J Neurosci 11:2382-2402.

Damasio AR, Yamada T, Damasio H, Corbet J, McKee J (1980) Central achromatopsia: behavioral, anatomical and physiologic aspects. Neurology 30:1064-1071. 
Dean P (1979) Visual cortex ablation and thresholds for successively presented stimuli in rhesus monkeys: II. Hue. Exp Brain Res 35:6983.

Desimone R, Schein S (1987) Visual properties of neurons in area V4 of the macaque: sensitivity to stimulus form. J Neurophysiol 57:835868.

Desimone R, Schein S, Moran J, Ungerleider I.G (1985) Contour, color and shape analysis beyond the striate cortex. Vision Res 25: $441-452$.

Haenny PE, Schiller PH (1988) State dependent activity in monkey visual cortex. I. Single cell activity in V1 and V4 on visual tasks. Exp Brain Res 69:225-244.

Haenny PE, Maunsell JHR, Schiller PH (1988) State dependent activity in monkey visual cortex. II. Retinal and extraretinal factors in V4. Exp Brain Res 69:245-259.

Heywood CA, Cowey A (1987) On the role of cortical area V4 in the discrimination of hue and pattern in macaque monkeys. J Neurosci 7:2601-2617.

Heywood CA, Gadotti A, Cowey A (1992) Cortical area V4 and its role in the perception of color. J Neurosci 12:4056-4065.

Julesz B (1981) Textons, the elements of texture perception, and their interactions. Nature 290:91-97.

Maunsell JHR, Newsome WT (1987) Visual processing in monkey extrastriate cortex. Annu Rev Neurosci 10:363-401.

Maunsell JHR, Sclar G, Nealey TA, DePriest DD (1991) Extraretinal representations in area V4 in the macaque monkey. Vis Neurosci 7:561-573

Meadows JC (1974) Disturbed perception of colors associated with localized cerebral lesions. Brain 97:615-632.

Moran J, Desimone R (1985) Selective attention gates visual processing in the extrastriate cortex. Science 229:782-784.

Motter BC (1992) Selective activation of V4 neurons in a color and orientation discrimination task. Invest Ophthalmol Vis Sci 33:1131.

Motter BC (1993) Focal attention produces spatially selective processing in visual cortical areas V1, V2 and V4 in the presence of competing stimuli. J Neurophysiol 70:909-919.

Motter BC (1994) Neural correlates of feature selective memory and pop-out in extrastriate area V4. J Ncurosci 14:2190-2199.

Motter BC, Mountcastle VB (1981) The functional properties of the light-sensitive neurons of the posterior parietal cortex studies in waking monkeys: foveal sparing and opponent vector organization. $J$ Neurosci 1:3-26.

Motter BC, Poggio GF (1984) Binocular fixation in the rhesus monkey: spatial and temporal characteristics. Exp Brain Res 54:304-314.
Mountcastle VB, Motter BC, Steinmetz MA, Sestokas AK (1987) Common and differential effects of attentive fixation on the excitability of parietal and prestriate (V4) cortical visual neurons in the macaque monkey. J Neurosci 7:2239-2255.

Petersen SE, Fox PT, Posner MI, Mintun M, Raichle ME (1988) Positron emission tomographic studies of the cortical anatomy of singleword processing. Nature 331:585-589.

Posner MI, Petersen SE, Fox PT, Raichle ME (1988) Localization of cognitive operations in the human brain. Science 240:1627-1631.

Regan D, Giaschi D, Sharpe JA, Hong XH (1992) Visual processing of motion-defined form: selective failure in patients with parietotemporal lesions. J Neurosci 12:2198-2210.

Schein SJ, Desimone R (1990) Spectral properties of V4 neurons in the macaque. J Neurosci 10:3369-3389.

Schiller PH, Lee K (1991) The role of the primate extrastriate area V4 in vision. Science 251:1251-1253.

Spitzer H, Desimone R, Moran J (1988) Increased attention enhances both behavioral and neuronal performance. Science 240:338-340.

Treisman A (1982) Perceptual grouping and attention in visual search for features and for objects. J Exp Psychol [Hum Percept] 8:194-214.

Treisman A, Gelade G (1980) A feature integration theory of attention. Cognit Psychol 12:97-136.

Treisman A, Schmidt H (1982) Illusory conjunctions in the perception of objects. Cognit Psychol 14:107-141.

Van Essen DC (1985) Functional organization of primate visual cortex. In: Cerebral cortex, Vol 3, Visual cortex (Peters A, Jones EG, eds). New York: Plenum.

Wild HM, Butler SR, Carden D, Kulikowski JJ (1985) Primate cortical area V4 important for color constancy but not wavelength discrimination. Nature 313:133-135.

Wolfe JM, Cave KR, Franzel SL (1989) Guided search: an alternative to the feature integration model for visual search. J Exp Psychol [Hum Percept] 15:419-433.

Zeki S (1973) Colour coding in rhesus monkey prestriate cortex. Brain Res 53:422-427.

Zeki S (1983) Colour coding in the cerebral cortex: the reaction of cells in monkey visual cortex to wavelengths and colours. Neuroscience 9:741-765.

Zeki S (1990) A century of cerebral achromatopsia. Brain 113:17211777.

Zeki S, Watson JDG, Lueck CJ, Friston KJ, Kennard C, Frackowiak RSJ (1991) A direct demonstration of functional specialization in human visual cortex. J Neurosci 11:641-649. 